\title{
Grid Enabled Non-rigid Registration with a Dense Transformation and a priori Information
}

\author{
Radu Stefanescu, Xavier Pennec, and Nicholas Ayache \\ INRIA Sophia, Epidaure, 2004 Rte des Lucioles, F-06902 Sophia-Antipolis Cedex \\ \{Radu.Stefanescu, Xavier.Pennec, Nicholas.Ayache\}@sophia.inria.fr \\ http://www-sop.inria.fr/epidaure/Epidaure-eng.html
}

\begin{abstract}
Multi-subject non-rigid registration algorithms using dense transformations often encounter cases where the transformation to be estimated requires a large spatial variability. In these cases, linear regularization methods are not sufficient. In this paper, we present an algorithm that uses a priori information about the nature of the images in order to find more adapted deformations. We also present a robustness improvement that gives higher weight to those points in the images that contain more information. Finally, a fast parallel implementation using networked personal computers is presented. Results show that our method can take into account the large variability of the inner brain structures. A parallel implementation allowed us to execute the registration algorithm in 5 minutes and future improvements will open the possibility of registering massive quantities of images.
\end{abstract}

\section{Introduction}

Unlike the mono-subject registration problem, which is relatively clear and corresponds to matching two instances of the same physical reality, the multi-subject registration problem is ill-posed. Indeed, the topology of the brain, the shape of the ventricles, the number and shape of the sulci vary strongly from one individual to another. Not only algorithms have to deal with the ambiguity of the structures to match, but they also have to take into account the large variability of the differences between the two brains. Often, brains are similar in several regions and entirely different in others.

However, multi-subject registration is an essential tool in neuro-science. Building and usage of brain atlases entirely depend on reliable multi-subject non-rigid registration. Also in the study of anatomic variability, shapes are compared by using non-rigid registration. Functional MRI gives the possibility of comparing the brain activity of different subjects, still using inter-subject nonrigid registration.

Recently, two main directions have emerged, depending on the type of transformation they use. By using a dense transformation, Cachier [6] proposed a pair-and-smooth algorithm which uses a gradient descent on a similarity criterion in order to estimate the match for each voxels and uniform Gaussian smoothing in order to regularize the transformation. It is therefore difficult for 
the algorithm to adapt to highly varying transformations. Hermosillo [5] used a variational approach to non-rigid registration. In order to deal with the high transformation variability problem, they use an approach borrowed from linear elasticity and proposed by Christensen [3].

Among sparse transformation algorithms, we would like to recall the approach proposed by Ferrant 4. An active surface is used to drive a finite element biomechanical model of the brain. The main advantage is the ability to take into account the various mechanical properties of the objects in the image. However, due to the limited resolution of the tetrahedrization imposed by computation time constraints, the algorithm is less adapted to the detection of small details. The algorithm is only capable to take advantage of a limited part of the information present in the images. Furthermore, since it depends on the physical modeling of deformation in elastic tissues, this algorithm is less adapted to multi-subject registration.

The purpose of our work is to combine the advantages of the two approaches presented above. We want to take advantage from the ability of the dense transformation algorithms to take into account the entire information present in the image, while still being capable to adapt the registration process to the nature of the objects present in the images.

Since the registration process is time consuming, we also address the problem of the execution time. From this point of view, our goal is to be able to run the algorithm in a sufficiently short time (typically one to two minutes) to be embedded in a more complex processing pipeline and allow for a possible interactivity between algorithm and user. Indeed, for multi-subject registration it is important that the user may correct or add additional a priori knowledge. We address this problem by using a parallel implementation on a cluster of networked personal computers. This type of parallel computing platform has the advantage of being readily available almost everywhere and may moreover be extended to the Grid, opening the possibility of registering and thus exploiting massive quantities of medical images.

\section{Method}

The registration process is described using a two term variational approach. At each step, the current transformation is estimated using two criteria: similarity and regularity. Through this article, we consider $J$ to be the source image, $I$ the target image and $U=\left(u_{1}, u_{2}, u_{3}\right)$ the displacement field that transforms $J$ into $I$, so that for each point $p, J(p+U(p))$ matches $I(p)$. If we denote Sim the similarity criterion that pushes the solution towards a better match and Reg the regularity criterion that makes the obtained deformation field regular, the registration process can be described using the variational formulation below:

$$
\frac{\partial u_{i}}{\partial t}=k \nabla \operatorname{Sim}(I, J \circ U)+(1-k) \nabla \operatorname{Reg}(U)
$$


where $k$ is the weight of the similarity criterion in the registration process. The meaning of the Sim and Reg criteria and the way they are linked together by using the weight $k$ will now be clarified.

\subsection{Matching}

In order to compute the displacement field $U$, a match is estimated for each point in the image $I$. In a mono-modal framework, this can be done by using a gradient descent on the Sum of Square Differences (SSD) as described in [6]:

$$
\operatorname{Sim}(I, J \circ U)=\sum_{p}(I(p)-(J \circ T)(p))^{2}
$$

A more robust method consists in also estimating the bias that affects the images. We have used the method proposed by Cachier and Pennec [2] which consists in using a more complex criterion: the local correlation coefficient. From a practical point of view, this amounts to iteratively estimate a smooth bias correction field and to optimize the SSD with a corrected image.

\subsection{Regularization}

The purpose of the regularization method is to allow for each voxel the selection of the degree of smoothness that we want to achieve. We have chosen to use the non-linear diffusion filter described by the following PDE:

$$
\frac{\partial u_{i}}{\partial t}=\nabla\left(\mathbf{d} \nabla u_{i}\right)
$$

If $\mathbf{d}$ is a scalar constant, then equation (3) describes a linear diffusion filter, which can be implemented using a convolution with a Gaussian. The bigger $\mathbf{d}$ is, the more important is the diffusion.

This formulation corresponds very well to our purpose of tolerating large deformations in certain areas and small deformations in others. It suffices to make $\mathbf{d}$ a scalar field and give it large values in area where we expect little deformation and small values in areas that have large ones. The $\mathbf{d}$ field can be estimated in several ways. The images may be segmented into regions and values of $\mathbf{d}$ can be assigned for each region. As we will show below, in the case of MRI$\mathrm{T} 1$ images, $\mathbf{d}$ can be estimated using a simple and almost automatic classification of the voxels in the images using an algorithm such as fuzzy k-means.

Finally, if dense diffusion information is available, $\mathbf{d}$ can be a dense diffusion tensor field. In this case, real world deformations can presumably be modeled with high precision.

\subsection{Trade-Off between Matching and Regularization: A Confidence-Based Approach}

An important problem of non-rigid registration algorithms with dense transformations is that matches are generally estimated for each voxel and all these 
matches contribute in an equal manner to the final deformation field. However, many of those matches only contribute false information, thereby making the registration process converge slower or even diverge. Experience shows that these "low confidence" points are the ones where signal to noise ratio is very low.

In our algorithm, it is the task of the weight factor $k$ to model the behavior in high confidence points versus the one if the confidence is low. We assign to each point $p$ in the image a confidence $k(p)$ in the interval $[0,1]$. Therefore, at each step, if the confidence in a point is high, the transformation in that point will be mainly driven by the similarity term. If the confidence is low, the transformation will mainly be computed through interpolation. In the current implementation, we use two ways of computing the confidence.

Static confidence. We use the assumption that high confidence points are the ones that lay in high variability neighborhoods of the source image. The confidence can therefore be a measure of the local variability of the source image, such as the local variance or the gradient. This type of measure is static, since it only has to be computed once, at the beginning of the algorithm. In our experiments, we used a normalized version of the norm of the gradient of the source image.

Dynamic confidence. The dynamic confidence attempts to assess the matches estimated by the similarity criterion. Another similarity criterion is computed in each voxel $p$ between $I(p)$ and $(J \circ T)(p)$. If the second criterion gives poor results, the voxel $p$ is assigned a low confidence. In our experiments, we used the squared local correlation coefficient which has the advantage of always belonging to the $[0,1]$ interval.

\section{$3 \quad$ Numerical Scheme}

The algorithm described above is reduced to solving an equation of the type $\frac{\partial u}{\partial t}=$ $\nabla \operatorname{Sim}+\nabla(\mathbf{d} \nabla u)$. The simplest way to solving such an equation is the explicit scheme $\frac{u^{t+\Delta t}-u^{t}}{\Delta t}=F^{t}+(\nabla(\mathbf{d} \nabla u))^{t}$, where $t$ is the time and $\Delta t$ is the time step. However, such an approach is very slow, since the time step has to be very small in order to avoid divergence. We therefore decided to use Weickert's Additive Operator Scheme (AOS) [8] in order to solve the $\frac{\partial u}{\partial t}=\nabla(\mathbf{d} \nabla u)$ equation and add at each step a fraction of the gradient of the similarity criterion.

Let us investigate first the resolution of the above equation in one dimension. If we compute the derivatives using finite differences and then reformulate the problem as a matrix vector multiplication, each step consists in solving $\frac{\mathbf{u}^{t+\Delta t}-\mathbf{u}^{t}}{\Delta t}=A \mathbf{u}^{t}$. Since we use finite differences, in each point $x, u^{t+\Delta t}(x)$ depends only on $u^{t}(x-1), u^{t}(x)$ and $u^{t}(x+1)$. Therefore, the matrix $A$ is tridiagonal. A better solution is to use the implicit scheme $\frac{\mathbf{u}^{t+\Delta t}-\mathbf{u}^{t}}{\Delta t}=A \mathbf{u}^{t+\Delta t}$ which is stable for all values of $\Delta t$. This means solving the equation $\mathbf{u}^{t+\Delta t}=$ $(I-\Delta t A)^{-1} \mathbf{u}^{t}$, with the matrix $I-\Delta t A$ also being tridiagonal. 
The inversion of a tridiagonal matrix can be achieved using the Thomas algorithm [7]. A LR decomposition is first done, then the matrix is inverted using one forward step and one backward step. Algorithmically, the LR decomposition and the forward and backward steps are first order recursive filters applied to the elements of the three non-null diagonals of the matrix.

In the three-dimensional case, a problem arises: the matrix to invert is no longer tridiagonal, which leads to a much higher computation time. However, if the filtering operator is separable, we can consider the operator $A$ to be the sum of his projection on the three axes $A=\sum_{d \in\{x, y, z\}} A_{d}$. Therefore

$$
\mathbf{u}^{t+\Delta t}=\left(I-\Delta t \sum_{d \in\{x, y, z\}} A_{d}\right)^{-1} \mathbf{u}^{t}
$$

In order to address the above-mentioned problem, Weickert 8] proved that the following approximation stands:

$$
\mathbf{u}^{t+\Delta t}=\left(I-\Delta t \sum_{d \in\{x, y, z\}} A_{d}\right)^{-1} \mathbf{u}^{t} \approx \frac{1}{3} \sum_{d \in\{x, y, z\}}\left(I-3 \Delta t A_{d}\right)^{-1} \mathbf{u}^{t}
$$

This reduces the $3 \mathrm{D}$ diffusion to three $1 \mathrm{D}$ ones, thus replacing the inversion of a non-tridiagonal matrix with three inversions of tridiagonal ones.

\section{Parallelization}

Even when using the AOS scheme, solving our PDE is a process lasting several tens of minutes. This is embarrassing for the user, especially if we want to include the registration into a longer processing chain which includes interactivity. We should then reach a mean computation time of a few minutes. Thus, we decided to implement the algorithm on a cluster of personal computers linked through a network. Our choice of the hardware platform was motivated by its high availability and its extremely low cost when compared to large multi-processor machines. The difficulty lays in the fact that the PC's that take part in the computation do not have direct access to each other's memory. Therefore explicit data transfers through the network are used in order to synchronize them.

At the beginning of the algorithm, the estimate of the transformation is cut into parallel thick slices and distributed to the available processors. Each processor computes the transformation for the voxels in its own slice. Each step of the algorithm can be decomposed in two logical parts: the estimate of the matches and the AOS step. The first step is fairly straightforward. The estimation of the match for one voxel is only dependent on the current value of the transformation in the voxel itself, so this step requires no communication between processes.

There are already existing implementations of the AOS on distributed memory parallel computers. Bruhn [1] proposes an implementation that requires clusters connected through high performance (hence expensive) networks. At each step, the image that is being filtered is redistributed, requiring all processors to communicate to each other. This implementation is not adapted to clusters of PC's connected through low cost networks. 
Since we cut our image in parallel slices and we filter independently in each directions, there are two directions for which all processes work entirely in parallel, without any need for communication. There is however one direction which is perpendicular on the slices and along which all the lines are distributed. The main difficulty in the parallelization of the AOS consists in the fact that the recursive nature of the Thomas does not allow for the parallel processing of a single line distributed on several nodes. We say that some processor $P$ precedes a processor $Q$ if in our algorithm we need to process the voxels of $P$ before processing the voxels of $Q$. We will also say that processor $Q$ "follows" $P$. Each time $P$ has finished processing its part of a line, it must send the computed value of the last voxel to $Q$ so that the latter process can begin processing the first voxel of its part of the line. Therefore, for each processed line, each processor waits for the data from its preceding processor, filters its part of the line and then sends the necessary data to the following machine.

The algorithm establishes a communication pattern that strongly resembles an industrial pipeline. One line is processed by a single processor at a time, but all the processes work simultaneously on different lines. At the very beginning of the execution, the processor that contains the beginning of the first line starts working, while other processors do nothing. Once it has finished processing its part of the first line, it passes the necessary data on to the second processor, while it begins processing its part of the second line. Hence, our pipeline takes a number of such small steps equal to the number of processors before being fully functional. The full acceleration is achieved if the number of lines in the image is much larger than the number of processors, which is generally true in clusters of PC's. This algorithm has the advantage if keeping communications to a minimum by not requiring any data redistribution.

\section{$5 \quad$ Results}

We evaluated the method by registering several couples of T1 MRI images coming from different subjects. We present one such couple in figures 1a) and 1(c). The image sizes are $256 \times 256 \times 124$. The images were affinely registered before the non-rigid registration. The normalized gradient of the source image was used as a static confidence measure. Voxels in the source image were classified based

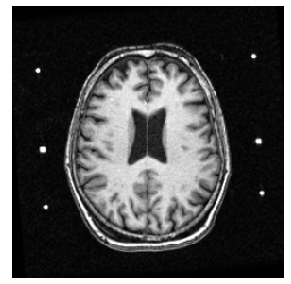

a) Source image

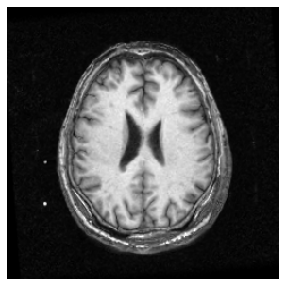

b) Registration result

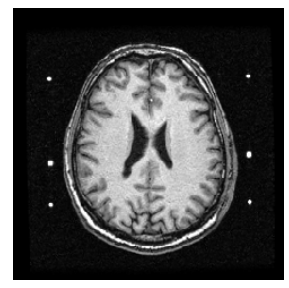

c) Reference image

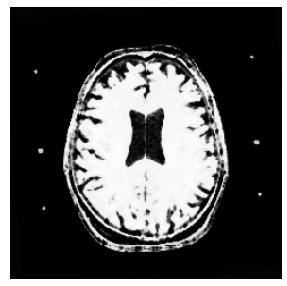

d) Stiffness

Fig. 1. The input images and the result of the registration. 


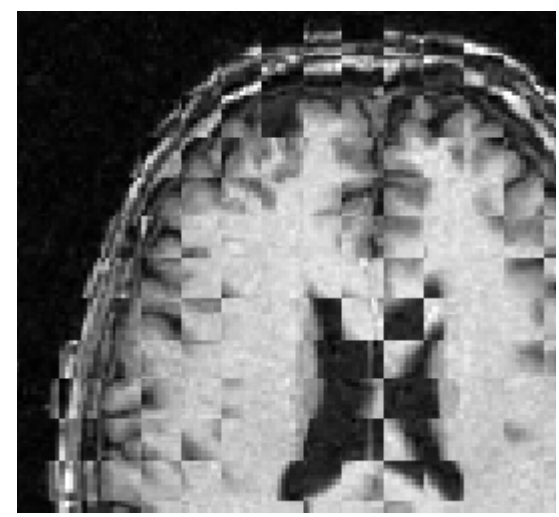

(a) Before registration

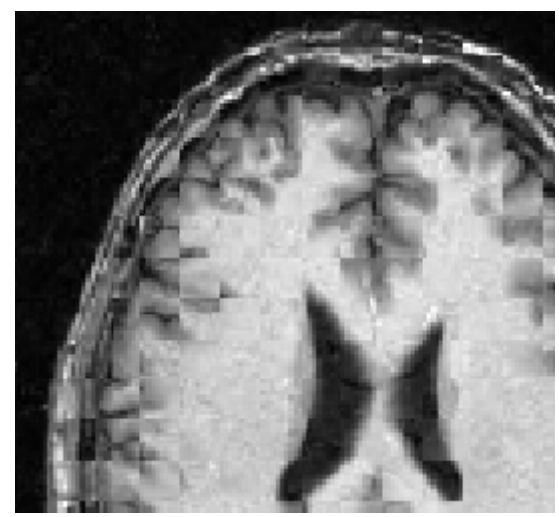

(b) After registration

Fig. 2. Mosaics of the two images before and after registration.

on their intensity into three classes: cerebro-spinal fluid, grey matter and white matter. The classification algorithm is a simple fuzzy k-means.

Figure 1(d) presents the probability that each voxel belong to either white or grey matter. This has been used as a measure of the material stiffness $\mathbf{d}$ in equation (3). A value close to 1 represents a stiff material, while a value close to 0 authorizes larger displacements. The classification algorithm classifies the skin as grey matter. This is not embarrassing, since we can safely assign the skin the same stiffness properties as the brain. The registration process converged in 40 iterations and took 5 minutes when executed in parallel on $152 \mathrm{GHz}$ Pentium IV PC's linked through a 1Gigabit/s network. The performance of the algorithm can however be improved by accelerating the convergence rate.

Figure 1(c) shows the result of the registration. Notice that assigning a very low stiffness (figure 1(d)) to the interior of the ventricles allowed them to follow very large deformations. Figure 2 shows a zoomed mosaic of the source and destination images before and after the registration. The algorithm was able to capture well the large changes in the shapes of the ventricles. An ideal registration would let the square model of the mosaic disappear. Remaining differences in the cortex give a measure of the difficulty of the problem: although the main sulci are present in every individual's brain at nearby locations, the overall sulcal map of the brain is very different.

\section{Conclusion}

We have presented a method for multi-subject non-rigid registration that uses a dense transformation. This method presents the advantages of the dense transformation (ability to take into account the entire information in the image), while 
being able to adapt the registration process to the nature of the object present in the images. This proved to be very helpful in the framework of multi-subject registration, which has to deal in the same time with high and low variability areas. Finally, a fast parallel implementation has been proposed.

Future work will investigate more appropriate ways of retrieving material informations, including the usage of other modalities and the incorporation of tissue anisotropy. We believe that improvement can also be made to the computation of the confidence coefficient. We expect that this will allow for more reliable matching information, thus allowing to reduce the number of iterations and finally decreasing the computation time. Future work will also be devoted to the difficult problem of validating this inter-patient registration algorithm.

Acknowledgments. This work was partially supported by the French region of Provence-Alpes-Côte d'Azur. The authors would like to thank Miguel Ángel González Ballester for providing the implementation of the fuzzy k-means algorithm and Guillaume Dugas-Phocion for the image presentation software. The images are courtesy of Pr. D. Dormont (Neuro-radiology Dept., Pitié-Salpétrière Hospital, Paris, France).

\section{References}

1. A. Bruhn, T. Jacob, M. Fischer, T. Kohlberger, J. Weickert, U. Brüning, and C. Schnörr. Designing 3-d nonlinear diffusion filters for high performance cluster computing. In LNCS, volume 2449, pages 396-399, 2002.

2. P. Cachier and X. Pennec. 3D non-rigid registration by gradient descent on a gaussian-windowed similarity measure using convolutions. In Proc. of IEEE Workshop on Mathematical Methods in Biomedical Image Analysis (MMBIA'00), pages 182-189, June 2000.

3. G. Christensen, M. Miller, and M. Vannier. A 3D deformable magnetic resonance textbook based on elasticity. In Proceedings of the American Association for Artificial Inteligence, Symposium: Applications of Computer Vision in Medical Image Processing, 1994.

4. M. Ferrant, S. Warfield, A. Nabavi, F. Jolesz, and R. Kikinis. Registration of 3D intraoperative $\mathrm{mr}$ images of the brain using a finite element biomechanical model. In Proceedings of MICCAI 2000, volume 1935 of LNCS, pages 19-28.

5. G. Hermosillo, C. Chefd'hotel, and O. Faugeras. Variational methods in multimodal image matching. IJCV, 50(3):329-343, 2002.

6. X. Pennec, P. Cachier, and N. Ayache. Understanding the "demon's algorithm": 3D non-rigid registration by gradient descent. In Proc. of MICCAI 1999, volume 1679 of $L N C S$, pages 597-605.

7. W.H. Press, B.P. Flannery, S.A. Teukolsky, and W.T. Vetterling. Numerical Recipes in $C$ : The Art of Scientific Computing. Cambridge University Press, 1993.

8. J. Weickert, B. Haar, and R. Viergever. Efficient and reliable schemes for nonlinear diffusion filtering. IEEE Trans. Image Proc., 7:398-410, 1998. 